\title{
A CAPACIDADE EXPLICATIVA (NÃO)CONDICIONAL DO BETA NAS AÇÕES DA EURONEXT LISBOA
}

THE (UN)CONDITIONAL EXPLANATORY ABILITY OF BETA IN EURONEXT LISBON STOCKS

Isabel Oliveira

Universidade Lusíada - Norte - Portugal isabel.m.m.oliveira@gmail.com

\author{
Submissão: 02 Nov 2018. Aceitação: 05/09/2019. \\ Publicação: 30 Set. 2019. Sistema de avaliação: Double \\ blind review. Universidade FUMEC / FACE, Belo Horizonte - \\ MG, Brasil. Editores: Prof. Dr. Mário Teixeira Reis Neto e Prof. \\ Dr. Cid Gonçalves Filho.
}

\section{RESUMO}

Este artigo analisa a capacidade explicativa, não condicional ou condicional, entre o risco medido pelo coeficiente beta e a rentabilidade das ações no mercado português. Este mercado pertence à Euronext que está fundida com a NYSE, e para o qual não existem estudos empíricos. A metodologia utilizada é similar à usada por Pettengill, Sundaram e Mathur (1995). Conclui-se que, o coeficiente beta, não condicional e condicional com a evolução do mercado, não é explicativo da rentabilidade das ações cotadas na Euronext Lisboa, assim como não existe um tradeoff risco/rentabilidade positivo. Constata-se apenas uma relação negativa, mas não consistente entre períodos, entre o beta e a rentabilidade quando o mercado está em baixa.

\section{PALAVRAS-CHAVE}

Capital asset pricing model. Rentabilidade. Beta. Beta condicional. Euronext Lisboa. 


\section{ABSTRACT}

This paper examines the explanatory ability and the unconditional or conditional relationship among risk, measured by the beta, and the return on securities in the Portuguese market.This is a market that belongs to Euronext, which is merged with the NYSE, for which there is no empirical studies. The methodology used is similar to the approach of Pettengill, Sundaram and Mathur (1995). One concludes that the unconditional and conditional beta with the market evolution does not explain return on securities in the Euronext Lisbon and that there is no positive risk/return tradeoff.There is only a negative relation, but it fails to be consistent between periods, between beta and return when the market is down.

\section{KEYWORDS}

Capital asset aricing aodel. Return. Beta. Conditional beta. Euronext Lisbon.

\section{INTRODUÇÃO}

O modelo CAPM considera um único fator de risco, o mercado, e estabelece uma relação linear e positiva entre a rentabilidade esperada de uma ação e o coeficiente beta. Alguns dos autores que confirmam a capacidade explicativa do modelo são Black, Jensen e Scholes (1972), Fama e MacBeth (1973) e Blume e Friend (1973). No entanto, estudos posteriores não corroboram a capacidade explicativa do coeficiente, de destacar Lakonishok e Shapiro (1986) e Reinganum (198I). Fama e French (1992) demonstram não existir uma relação sistemática entre o coeficiente beta e a rentabilidade das ações no mercado dos EUA. Estes autores marcam o início da controvérsia sobre a validade do CAPM, quer na relação beta/rentabilidade, quer na capacidade explicativa do coeficiente beta.

Pettengill, Sundaram e Mathur (1995) argumentam que o modelo CAPM é estimado com rentabilidades efetivas como proxies de rentabilidades esperadas e, é provável que a rentabilidade em excesso de mercado (diferença entre a rentabilidade de mercado e a rentabilidade do ativo isento de risco) seja negativa em alguns períodos. Estes adaptam a metodologia de Fama e MacBeth (1973), separando períodos de rentabilidade em excesso de mercado positivos (mercado em alta) e negativos (mercado em baixa). Pettengill, Sundaram e Mathur (1995) ao contrário dos estudos anteriores, constatam uma relação significativa e condicional entre beta e rentabilidade, positiva quando o mercado está em alta e negativa quando o mercado está em baixa. Concluem que o beta deve continuar a ser uma medida adequada de risco. Após este estudo vários autores confirmam esta relação condicional entre beta e rentabilidade, de destacar, Fletcher (2000), Hung, Shackleton e Xu (2004) e Jianhua e Wihlborg (20 I 0). No entanto, Lewellen e Nagel (2006) e Durand, Lang e Ng (20 I I) constatam que 0 beta condicional apresenta uma capacidade explicativa fraca tal como o beta não condicional.

O objetivo deste artigo é analisar a exis- 
tência de uma relação entre o coeficiente beta do CAPM e a rentabilidade das ações cotadas na Euronext Lisboa. Para tal, analisase a existência de uma relação, não condicional e positiva ou, condicional com a evolução do mercado, positiva para mercado em alta e negativa para mercado em baixa. O segundo objetivo é analisar a existência de um tradeoff risco/rentabilidade positivo.

Existem vários fatores que diferenciam esta análise dos estudos existentes em termos de mercado e características deste, assim como a metodologia utilizada.

Primeiro, os estudos empíricos ao mercado português são praticamente inexistentes e quando este mercado é analisado, não o é isoladamente, mas é incluído numa amostra em termos de um mercado global.

Segundo, a pertença do mercado português na Euronext e a fusão deste mercado na NYSE, torna um dos maiores mercados bolsistas internacionais. Acrescenta-se ainda que, a globalização econômica e consequente integração entre os mercados, torna possíveis estratégias de investimento diversificadas internacionalmente.

Terceiro, este mercado apresenta características que o diferenciam dos mercados analisados. Contrariamente às empresas analisadas em estudos anteriores, em Portugal, predominam empresas de pequena dimensão. Em média os coeficientes betas das empresas de menor dimensão são superiores relativamente aos das restantes empresas. Assim, se as empresas portuguesas apresentam coeficientes betas maiores, as oscilações de rentabilidade também são superiores e podem apresentar comportamento diferente das ações de empresas de maior dimensão.

Quarto, as carteiras utilizadas nesta análise são constituídas anualmente, permi- tindo que estas sejam ajustadas durante $o$ período da amostra. Metodologia que apresenta vantagem comparativamente aos estudos que utilizam carteiras que são mantidas ao longo de todo o período em análise (Pettengill; Sundaram; Mathur, 1995).

$\mathrm{O}$ artigo está organizado da seguinte forma: Na seção seguinte é apresentada a revisão da literatura. Posteriormente é apresentada a metodologia e a amostra utilizada. Em seguida são apresentados e analisados os resultados empíricos. As conclusões finais são apresentadas na última seção.

\section{REVISÃO DA LITERATURA}

Principalmente a partir da década de 1980, os estudos empíricos à validade do modelo CAPM, colocam em causa a capacidade explicativa do coeficiente beta. Tinic e West (1984) rejeitam a validade do CAPM com base na inconsistência temporal do modelo. O tradeoff risco/rentabilidade, é verificado apenas no mês de janeiro, por ser o único mês em todo o período da amostra que a rentabilidade em excesso de mercado é positiva.

Pettengill, Sundaram e Mathur (1995) argumentam que é necessário existir três pressupostos para o modelo CAPM ser válido. Primeiro, a rentabilidade esperada para o mercado deve ser superior à rentabilidade do ativo isento de risco, caso contrário, todos os indivíduos investem neste ativo. Segundo, se os investidores tivessem a certeza que a rentabilidade de mercado é superior à rentabilidade de um ativo isento de risco, então nenhum indivíduo investia neste ativo. Terceiro, carteiras com coeficientes betas mais elevados, apresentam risco mais elevado e devem ter rentabilidades mais elevadas.

O modelo assume assim duas hipóte- 
ses: (i) a existência de um tradeoff risco/ rentabilidade positivo e (ii) carteiras com coeficientes betas mais elevados apresentam rentabilidades esperadas mais elevadas que as carteiras com este coeficiente mais baixo. Estas hipóteses só se verificam se a rentabilidade de mercado for superior à rentabilidade do ativo isento de risco $\left(R m_{t}>R f_{t}\right.$, rentabilidade em excesso de mercado positiva ou mercado em alta). No caso da rentabilidade de mercado ser inferior à rentabilidade do ativo isento de risco $\left(R_{\mathrm{t}}<\mathrm{Rf}_{\mathrm{t}}\right.$, rentabilidade em excesso de mercado negativa, ou mercado em baixa), o prêmio de risco é negativo $\left(\left[R m_{t}-R f_{t}\right]<0\right)$. Consequentemente existe uma relação inversa entre o coeficiente beta e a rentabilidade esperada, pelo que, carteiras com betas elevados proporcionam rentabilidades esperadas mais baixas que carteiras com betas baixos. Pettengill, Sundaram e Mathur (I995) confirmam a relação condicional, positiva para mercado em alta e negativa para mercado em baixa, entre o coeficiente beta e a rentabilidade em ações cotadas nos EUA, no período de 1936 a 1990.

Vários estudos com amostras de diferentes mercados confirmam os resultados de Pettengill, Sundaram e Mathur (1995), de destacar: Crombez e Vennet (2000) no mercado Belga, Elsa, El-Shaer e Theissen (2003) no mercado Alemão, Theriou, Aggelidis, Maditinos et al. (2010) no mercado da Grécia, Hung, Shackleton e Xu (2004), Cotter, O'Sullivan e Rossi (2015) e Morelli (20 I I) no Reino Unido, Terregrossa e Eraslan (2016) no mercado Turco, Fletcher (2000) em dezoito mercados desenvolvidos, Jagannathan e Wang (1996) e Xiao (2016) no mercado dos EUA, Jianhua e Wihlborg (20l0) em seis mercados emergentes da Europa, Sandoval e Saens (2004) em quatro mercados da América Latina, Tang e Shum (2004) no mercado de Singapura, Nimal e Fernando (20l3) no mercado do Japão e Sri Lanka e Anuradha (20 I I) no mercado do Sri Lanka.

No entanto, Lewellen e Nagel (2006) constatam que o beta condicional apresenta uma capacidade explicativa fraca tal como o beta não condicional. Durand, Lan e $\mathrm{Ng}$ (20ll) utilizam diferentes medidas de rentabilidade em excesso de mercado a uma amostra de mercados emergentes do Pacífico. Constatam que em apenas uma das medidas existe evidência estatística para apoiar o coeficiente beta condicional. Abdullah,Al-Jafari, Tai e Ahmad (20 I I) em uma amostra de ações do Kuwait concluem que o coeficiente beta não apresenta poder explicativo em situações de rentabilidade em excesso de mercado negativa.

\section{METODOLOGIA E AMOSTRA}

Para analisar a capacidade explicativa do coeficiente beta do CAPM, não condicional e condicional, na rentabilidade das ações cotadas na Euronext Lisboa, são enunciadas as seguintes hipóteses de pesquisa, com os dados agregados em carteiras:

HI: Existe uma relação sistemática, não condicional e positiva, entre o coeficiente beta e a rentabilidade das ações.

H2: Existe uma relação sistemática, mas condicional entre o coeficiente beta e a rentabilidade, positiva quando o mercado está em alta e negativa quando o mercado está em baixa.

H3: Existe um tradeoff risco/rentabilidade positivo. A amostra utilizada é constiz tuída por todas as ações cotadas no mercado português, Euronext Lisboa, exceto as pertencentes ao setor financeiro, por apresentarem um leverage elevado entre janei- 
ro/l999 (ano da entrada da moeda única) e dezembro/2016. Apenas são incluídas as ações que apresentem cotações mensais consecutivas não constantes durante um período mínimo de vinte e quatro meses, por ser o período mínimo aceitável para estimar os parâmetros dos modelos e também por ser considerado um critério de liquidez das ações. Desta seleção resultam 86 ações cotadas na Euronext Lisboa.

A metodologia utilizada, adaptada da metodologia de Pettengill, Sundaram e Mathur (1995), é definida em três fases a que correspondem três subperíodos da amostra, de acordo com a Tabela I.

São dois os critérios considerados para a divisão da amostra. Primeiro, nas duas primeiras fases é considerado um número idêntico de observações mensais. Segundo é incluído em cada uma das fases um número mínimo de observações que permita obter estimativas fiáveis para os parâmetros dos modelos. As ações incluídas na amostra poderão não apresentar a totalidade das observações ao longo dos dezoito anos da amostra. Na segunda e terceira fase é permitido a saída de ações posteriormente ao primeiro período da amostra, mas não se considera a entrada de novas ações.

Na primeira fase, com o primeiro período da amostra, são estimados os coeficientes betas das ações, com base no modelo de mercado, representado pela seguinte equação:

$$
R_{i, t}=\alpha_{i}+\beta_{i} R m_{t}+\varepsilon_{i, t}
$$

Em que: $R_{i, t}$ é a rentabilidade da ação i no mês $t, R_{t}$ é a rentabilidade da carteira de mercado no mês t, e $\varepsilon_{i, t}$ é uma perturbação aleatória do modelo referente à ação i no mês t. $O$ coeficiente $\beta_{\text {i }}$ representa o beta da ação i. A rentabilidade de mercado é a rentabilidade média simples de todas as ações incluídas na amostra. Assume-se que os erros da regressão são independentes e identicamente distribuídos, com média zero e matriz de covariâncias estacionárias.

As carteiras que servem de base à estimação dos modelos são constituídas anualmente, com base na ordenação crescente dos betas das ações, estimados com 60 rentabilidades mensais, deslizando um ano no período temporal e deixando cair o primeiro ano.A vantagem da constituição anual de carteiras é permitir que estas sejam ajustadas anualmente durante o período da amostra e corrigidos das eventuais saídas de ações. Na metodologia de Pettengill, Sundaram e Mathur (1995) as carteiras são constituídas com o primeiro período da amostra e mantidas na totalidade do período em análise.

A amostra utilizada nesta investigação é mais reduzida que a utilizada por outros estudos empíricos. Para ultrapassar o inconveniente de carteiras pouco diversificadas, opta-se por constituir nove carteiras. As ações com betas mais baixos são incluídas na primeira carteira, e assim sucessivamente até à última carteira, constituída pelas ações com betas mais elevados.

$\mathrm{Na}$ segunda fase, com o segundo perí-

TABELA 1 - Divisão da Amostra em Três Períodos

\begin{tabular}{l|c|c|c}
\hline \multirow{2}{*}{ Períodos/Fases: } & $1^{\mathrm{a}}$ Fase & $2^{\mathrm{a}}$ Fase & $3^{\mathrm{a}}$ Fase \\
\cline { 2 - 4 } & Constituição das Carteiras & Estimação dos Betas das Carteiras & Análise dos Modelos \\
\hline Períodos & $01 / 1999$ a $12 / 2003$ & $01 / 2004$ a $12 / 2008$ & $01 / 2009$ a $12 / 2016$ \\
\hline N. ${ }^{\circ}$ observações & 60 meses & 60 meses & 96 meses \\
\hline
\end{tabular}

Fonte: 
odo da amostra, estima-se os coeficientes betas das carteiras (constituídas na primeira fase) através do modelo de mercado representado pela equação (I). É utilizada a metodologia de janela deslizante. Os betas das carteiras são estimados sempre com base em 60 observações mensais, deslizase um mês no período temporal e deixa-se cair a primeira observação. A rentabilidade das carteiras é uma média simples das rentabilidades das ações que compõem cada uma das carteiras.

$\mathrm{Na}$ terceira e última fase, as estimativas dos betas das carteiras, obtidos de regressões temporais na segunda fase da amostra, são utilizados como variáveis explicativas das regressões seccionais dos modelos a testar, representadas pelas seguintes equações:

$$
\begin{aligned}
& R_{j, t}=\gamma_{0, t}+\gamma_{l, t} \beta_{j, t-1}+\varepsilon_{j, t} \\
& R_{j, t}=\gamma_{0, t}+\gamma_{2, t} D_{t} \beta_{j, t-1}+\gamma_{3, t}\left(I-D_{t}\right) \beta_{j, t-1}+
\end{aligned}
$$$$
\varepsilon_{j, t}
$$

Em que: $R_{i, t}$ é a rentabilidade da carteira j no mês $t, \beta_{j, t-1}$ é o coeficiente beta da carteira $\mathrm{j}$ estimado no período anterior $(\mathrm{t}-\mathrm{I})$, $\varepsilon_{j t}$ é uma perturbação aleatória do modelo referente à carteira $\mathrm{j}$ no mês $\mathrm{t}$ e os parâmetros dos modelos são $Y_{0, t^{\prime}} V_{l, t^{\prime}}, V_{2, t}$ e, $V_{3, t^{*}}$ $A$ variável dummy, $D_{t}$, assume o valor de: I se o mercado está em alta, isto é, a rentabilidade em excesso de mercado positiva $\left(\left[R m_{t}-R f_{t}\right]>0\right)$ e, o valor de 0 se o mercado está em baixa, isto é, a rentabilidade em excesso de mercado negativa $\left(\left[\mathrm{Rm}_{\mathrm{t}}-\mathrm{Rf}_{\mathrm{t}}\right]\right.$ $<0)$.

A equação (2), apresenta $\circ$ beta não condicional e, se for verificada a primeira hipótese, o valor do parâmetro $\gamma_{l}$, terá de ser positivo e estatisticamente diferente de zero. Assim, é testada a seguinte hipótese ao parâmetro do modelo:

$$
\begin{aligned}
\mathrm{H}_{0}: \overline{\mathrm{Y}}_{1} & =0 \\
\mathrm{H}_{1} & : \overline{\mathrm{Y}}_{1}>0
\end{aligned}
$$

A equação (3), apresenta o beta condicional e, se for verificada a segunda hipótese existem alguns sinais esperados para os coeficientes do modelo. $O$ coeficiente $Y_{2}$ é a estimativa do prêmio de risco quando o mercado está em alta, espera-se que seja positivo e estatisticamente diferente de zero. De igual modo, $\mathrm{V}_{3}$ é a estimativa do prêmio de risco quando o mercado está em baixa, espera-se que seja negativo e estatisticamente diferente de zero.Assim, são testadas as seguintes hipóteses propostas por Pettengill, Sundaram e Mathur (1995):

$$
\begin{array}{lll}
\mathrm{H}_{0}: \overline{\mathrm{Y}}_{2}=0 & ; & \mathrm{H}_{0}: \overline{\mathrm{Y}}_{3}=0 \\
\mathrm{H}_{1}: \overline{\mathrm{Y}}_{2}>0 & ; & \mathrm{H}_{1}: \overline{\mathrm{Y}}_{3}<0
\end{array}
$$

$\mathrm{Na}$ análise da terceira hipótese de pesquisa, a existência de um tradeoff risco/ rentabilidade positivo, terão de se verificar duas condições: (I) a rentabilidade em excesso de mercado terá de ser positiva e, (2) os sinais esperados para os coeficientes diferem com a evolução do mercado, pelo que o prêmio de risco deve ser simétrico para mercado em alta e para mercado em baixa. Esta condição é testada através da seguinte hipótese:

$$
\begin{gathered}
\mathrm{H}_{0}: \overline{\mathrm{Y}}_{2}-\overline{\mathrm{Y}}_{3}=0 \\
\mathrm{H}_{1}: \overline{\mathrm{Y}}_{2}-\overline{\mathrm{Y}}_{3} \neq 0
\end{gathered}
$$

No teste de simetria é utilizado o valor simétrico de $Y_{3}$.

As estimativas de $\bar{Y}_{1}, \bar{Y}_{2}$ e $\bar{Y}_{3}$ são as médias dos valores das estimativas dos coeficientes de $Y_{1, t} V_{2, t}$ e $V_{3, t}$ obtidos em cada uma das regressões seccionais.

\section{RESULTADOS EMPÍRICOS}

Relação (Não)Condicional entre Beta e Rentabilidade

O objetivo desta secção é analisar o poder explicativo do coeficiente beta e a sua 
relação com a rentabilidade: na equação (2) a relação não condicional, sistemática e positiva e, na equação (3) a relação condicional, positiva quando o mercado está em alta e negativa quando o mercado está em baixa. Análise realizada com o terceiro período da amostra, de janeiro/2009 a dezembro/2016, perfazendo noventa e seis meses. Para analisar se os resultados são consistentes ao longo do período em análise, o terceiro período da amostra é divido em dois subperíodos, um e dois, de janeiro/2009 a dezembro/20I 2 e de janeiro/20I3 a dezembro/20I6, perfazendo quarenta e oito observações mensais em cada um dos dois subperíodos.

As equações apresentam como variável explicativa os coeficientes betas que são estimados com base nas 60 observações mensais anteriores. Os parâmetros das equações (2) e (3) são os valores médios dos parâmetros obtidos nas regressões seccionais. $\mathrm{Na}$ estimação dos modelos é utilizado o método dos mínimos quadrados ordinários e o nível de significância estatística ( $p$-level) utilizado nos testes estatísticos é de $5 \%$.

$\mathrm{Na}$ Tabela 2 são apresentados os resul- tados das regressões seccionais da equação (2) para as nove carteiras constituídas com base nos coeficientes betas das ações individuais.

As médias estatisticamente significativas, a um nível de significância de $5 \%$, estão assinaladas a negrito.

A Tabela 2 evidencia que o coeficiente beta não é estatisticamente significativo para explicar a rentabilidade das carteiras. A hipótese nula, a média de $\gamma_{1}$ é estatisticamente igual a zero, não é rejeitada nos três períodos em análise. Não se verifica a existência de uma relação entre o risco, medido pelo coeficiente beta e a rentabilidade das carteiras para as ações cotadas no mercado português.

A constante do modelo, o coeficiente $\gamma_{0}$, que representa a rentabilidade de um ativo com beta zero, evidência que a média deste coeficiente é igual a zero. A média da estimativa de $\mathrm{Y}_{0}$ é negativa no período dois, o que pode ser interpretado como uma rentabilidade negativa fixa para as carteiras. Este resultado também é encontrado em alguns estudos, como Fletcher (2000) e Isakov (1999).

TABELA 2 - Modelo com Beta Não Condicional

\begin{tabular}{|c|c|c|c|c|}
\hline \multirow{2}{*}{ Período: } & & \multicolumn{3}{|c|}{ Média das Regressões: $R_{i, t}=\gamma_{0, t}+\gamma_{1, t} \beta_{i}+e_{i, t}$} \\
\hline & & $\mathrm{Y}_{0}$ & $Y_{1}$ & $\mathrm{R}^{2}$ Ajustado \\
\hline \multirow{4}{*}{$\begin{array}{l}\text { Período Total } \\
\text { (01/2009 a 12/2016) }\end{array}$} & Mean & 0,0004 & 0,0117 & \multirow{4}{*}{0,1407} \\
\hline & & & & \\
\hline & T-statistic & 0,0175 & 0,5732 & \\
\hline & $P$-value & 0,9861 & 0,5681 & \\
\hline \multirow{4}{*}{$\begin{array}{l}\text { Período } 1 \\
\text { (01/2009 a 12/2012) }\end{array}$} & Mean & 0,0257 & $-0,0248$ & \multirow{4}{*}{0,0635} \\
\hline & & & & \\
\hline & T-statistic & 0,8700 & $-1,1017$ & \\
\hline & $P$-value & 0,3894 & 0,2770 & \\
\hline \multirow{3}{*}{$\begin{array}{l}\text { Período } 2 \\
\text { (01/2013 a 12/2016) }\end{array}$} & Mean & $-0,0249$ & 0,0482 & \multirow{3}{*}{0,2179} \\
\hline & T-statistic & $-0,8666$ & 1,4427 & \\
\hline & $P$-value & 0,3912 & 0,1567 & \\
\hline
\end{tabular}

Fonte: 
As médias dos coeficientes de determinação ajustados são baixas, variam entre $6,4 \%$ (período I) e $21,8 \%$ (período 2), o que confirmam o anteriormente exposto.

Conclui-se que não é verificada a primeira hipótese de pesquisa, resultados que são consistentes com outros estudos (Fletcher, 2000, Theriou;Aggelidis; Maditinos et al., 20l0).

A Tabela 3 apresenta os resultados das regressões seccionais da equação (3) para as nove carteiras constituídas com base nos coeficientes betas das ações individuais. A rentabilidade mensal em excesso de mercado é a diferença entre a rentabilidade de mercado e a rentabilidade do ativo isento de risco em cada um dos meses. Para a rentabilidade do ativo isento de risco é utilizada a taxa mensal das obrigações da Alemanha há dez anos.

As médias estatisticamente significativas, a um nível de significância de $5 \%$, estão assinaladas em negrito.

Os resultados da Tabela 3 evidenciam que quando o mercado está em baixa, a média da estimativa de $\gamma_{3}$ é negativa, existe um prêmio de risco negativo para as carteiras. $\bigcirc$ valor médio deste coeficiente é estatisticamente diferente de zero no período total e um, nestes períodos existe uma relação sistemática e negativa entre o coeficiente beta e a rentabilidade. Relação que não é verificada no período dois, pelo que não existe consistência temporal. Quando o mercado está em alta, não se rejeita a hipótese nula, não é confirmada a relação sistemática e positiva entre o coeficiente beta e a rentabilidade, resultado que é consistente entre períodos.

A média da estimativa de $Y_{0}$ é negativa para o mercado em baixa e positiva para o mercado em alta, exceto no período dois, que é sempre negativa. As médias dos coeficientes de determinação ajustado são baixas, variam entre $3,4 \%$ (no período um para mercado em alta) e $24,7 \%$ (no período dois para mercado em alta), justificando o anteriormente exposto.

Conclui-se que não é verificada a segunda hipótese de pesquisa. Apenas é verificada uma relação negativa entre beta/rentabilidade quando o mercado está em baixa, mas esta relação não é consistente. Resultados que não estão de acordo com alguns estudos para o mercado da Europa (Cot-

TABELA 3 - Modelo com Beta Condicional para Mercado em Alta e Mercado em Baixa

\begin{tabular}{|c|c|c|c|c|c|c|c|}
\hline \multirow{3}{*}{ Período: } & & \multicolumn{6}{|c|}{ Média das Regressões: $R_{j, t}=\gamma_{0}+\gamma_{2} D_{t} \beta_{j-1}+\gamma_{3}\left(1-D_{t}\right) \beta_{j-1}+\varepsilon_{j, t}$} \\
\hline & & \multicolumn{3}{|c|}{ Mercado em Alta } & \multicolumn{3}{|c|}{ Mercado em Baixa } \\
\hline & & $\mathrm{Y}_{0}$ & $\mathrm{Y}_{2}$ & $\mathrm{R}^{2}$ Ajust. & $\mathrm{Y}_{0}$ & $\mathrm{Y}_{3}$ & $\mathrm{R}^{2}$ Ajust. \\
\hline \multirow{4}{*}{$\begin{array}{l}\text { Período Total } \\
(01 / 2009 \text { a } 12 / 2016)\end{array}$} & Mean & 0,0053 & 0,0578 & & $-0,0046$ & $-0,0344$ & \\
\hline & & & 15027 & 01552 & & 20217 & 1261 \\
\hline & & & & & & & \\
\hline & $P$-value & 0,8955 & 0,1403 & & 0,6304 & 0,0019 & \\
\hline \multirow{3}{*}{$\begin{array}{l}\text { Período } 1 \\
\text { (01/2009 a 12/2012) }\end{array}$} & Mean & 0,0634 & $-0,0060$ & & $-0,0027$ & $-0,0389$ & \\
\hline & T-statistic & 0.9461 & -0.1197 & 0.0338 & -0.2244 & -28904 & 00857 \\
\hline & $P$-value & 0,3573 & 0,9061 & & 0,8244 & 0,0083 & \\
\hline \multirow{3}{*}{$\begin{array}{l}\text { Período } 2 \\
(01 / 2013 \text { a 12/2016) }\end{array}$} & Mean & $-0,0382$ & 0,1057 & & $-0,0072$ & $-0,0284$ & \\
\hline & T-statistic & $-0,7763$ & 1,9293 & 0,2465 & $-0,4517$ & $-1,7179$ & 0,1798 \\
\hline & $P$-value & 0,4455 & 0,0661 & & 0,6572 & 0,1040 & \\
\hline
\end{tabular}

Fonte: 
ter; O'Sullivan; Rossi, 20I5, Morelli, 20II, Theriou; Aggelidis; Maditinos et al., 20l0).

Para analisar a existência de sazonalidade, o terceiro período da amostra é dividido em dois, para o mês de janeiro e para os restantes onze meses. A Tabela 4 apresenta os resultados das regressões seccionais das equações (2) e (3) com o terceiro período da amostra dividido em dois subperíodos, o mês de janeiro e os restantes onze meses.

As médias estatisticamente significativas, a um nível de significância de $5 \%$, estão assinaladas em negrito.

Os valores das regressões da Tabela 4 confirmam os resultados anteriormente encontrados. A relação, não condicional e condicional, entre beta/rentabilidade não existe nas ações cotadas na Euronext Lisboa. Existe uma relação negativa, mas inconsistente entre períodos, quando o mercado está em baixa. Relação não existente no mês de janeiro, mas existente nos onze meses restantes. A existência de sazonalidade no mês de janeiro, constatada em alguns estudos, nomeadamente Tinic e West (1984) e Pettengill, Sundaram e Mathur (1995) não é confirmada na amostra de ações cotadas no mercado Português.

\section{TABELA 4 - Modelo com Beta Não Condicional e Condicional por Meses}

\begin{tabular}{|c|c|c|c|c|c|c|c|}
\hline \multirow{2}{*}{ Período: } & \multicolumn{7}{|c|}{ Média das Regressões: $R_{i, t}=\gamma_{0, t}+\mathrm{Y}_{1, t} \beta_{i}+e_{i, t}$} \\
\hline & & & \multicolumn{2}{|c|}{$\mathrm{Y}_{0}$} & \multicolumn{2}{|c|}{$\mathrm{Y}_{1}$} & $R^{2}$ Ajustado \\
\hline \multirow{3}{*}{ Período Total } & Mean & & \multicolumn{2}{|c|}{0,0004} & \multicolumn{2}{|c|}{0,0117} & \\
\hline & T-statistic & & \multicolumn{2}{|c|}{0,0175} & \multicolumn{2}{|c|}{0,5732} & 0,1407 \\
\hline & $P$-value & & \multicolumn{2}{|c|}{0,9861} & \multicolumn{2}{|c|}{0,5681} & \\
\hline \multirow{3}{*}{ Mês: Janeiro } & Mean & & \multicolumn{2}{|c|}{$-0,0074$} & \multicolumn{2}{|c|}{0,0484} & \\
\hline & T-statistic & & \multicolumn{2}{|c|}{$-0,1531$} & \multicolumn{2}{|c|}{0,9616} & 0,1095 \\
\hline & $P$-value & & \multicolumn{2}{|c|}{0,8833} & \multicolumn{2}{|c|}{0,3734} & \\
\hline \multirow{3}{*}{ Meses: Fev. a Dez. } & Mean & & \multicolumn{2}{|c|}{0,0011} & \multicolumn{2}{|c|}{0,0084} & \\
\hline & T-statistic & & \multicolumn{2}{|c|}{0,0481} & \multicolumn{2}{|c|}{0,3833} & 0,1435 \\
\hline & $P$-value & & \multicolumn{2}{|c|}{0,9627} & \multicolumn{2}{|c|}{0,7026} & \\
\hline \multirow{3}{*}{ Período: } & & \multicolumn{6}{|c|}{ Média das Regressões: $R j, t=\gamma 0+\gamma 2 D t \beta j-1+\gamma 3(1-D t) \beta j-1+\varepsilon j, t$} \\
\hline & & \multicolumn{3}{|c|}{ Mercado em Alta } & \multicolumn{3}{|c|}{ Mercado em Baixa } \\
\hline & & $\mathrm{V}_{0}$ & $\mathrm{Y}_{2}$ & $\mathrm{R}^{2}$ Ajust. & $\mathrm{Y}_{0}$ & $\mathrm{Y}_{3}$ & $\mathrm{R}^{2}$ Ajust. \\
\hline \multirow{3}{*}{ Período Total } & Mean & 0,0053 & 0,0578 & & $-0,0046$ & $-0,0344$ & \\
\hline & T-statistic & 0,1321 & 1,5037 & 0,1553 & 0,4848 & $-3,3217$ & 0,1261 \\
\hline & $P$-value & 0,8955 & 0,1403 & & 0,6304 & 0,0019 & \\
\hline \multirow{3}{*}{ Mês: Janeiro } & Mean & 0,0014 & 0,0750 & & $-0,0295$ & $-0,0181$ & \\
\hline & T-statistic & 0,0208 & 1,0955 & 0,1870 & $-2,7793$ & $-1,5981$ & 0,3807 \\
\hline & $P$-value & 0,9844 & 0,3348 & & 0,2199 & 0,3560 & \\
\hline \multirow{3}{*}{ Meses: Fev. a Dez. } & Mean & 0,0058 & 0,0555 & & $-0,0034$ & $-0,0352$ & \\
\hline & T-statistic & 0,1299 & 1,2939 & 0,1510 & $-0,3388$ & $-3,2436$ & 0,1366 \\
\hline & $P$-value & 0,8973 & 0,2039 & & 0,7366 & 0,0024 & \\
\hline
\end{tabular}

Fonte: 


\section{O TRADEOFF RISCOI RENTABILIDADE POSITIVO}

Para existir um tradeoff risco/rentabilidade positivo, tem de se verificar as duas condições enunciadas na definição da metodologia.

A Tabela 5 apresenta as estatísticas descritivas da rentabilidade em excesso de mercado no período total (0I/2009 a I2/2016) e desagregado nos dois períodos, um e dois.

A rentabilidade média em excesso de mercado é positiva no período total e no período dois, mas negativa no período um. Neste período, o mercado não proporciona uma recompensa positiva pelo risco de mercado, pelo que os investidores não são remunerados, positivamente, pelo risco que assumem. Nos períodos, total e um, o $p$-value evidencia que a rentabilidade média em excesso de mercado é igual a zero. Hipótese que é rejeitada no período dois.

A segunda condição para existir um tradeoff risco/rentabilidade positivo, requer que o prêmio de risco seja simétrico para mercado em alta e em baixa, daí que as médias das estimativas de $Y_{2}$ e $Y_{3}$ da equação (3) (Tabela 3) devem apresentar os sinais esperados, positivo para mercado em alta $\left(\mathrm{Y}_{2}\right)$ e negativo para mercado em baixa $\left(\mathrm{Y}_{3}\right)$. No período um, não é verificada esta condição, a média de cada um dos dois coeficientes é negativa, qualquer que seja a evolução de mercado.

Os resultados do teste de hipótese à simetria dos coeficientes $\left(H_{0}: \bar{Y}_{2}-\bar{Y}_{3}=0\right)$ são apresentados na Tabela 6 .

Constata-se na Tabela 6, um nível de significância estatística de $5 \%$, que é rejeitada a hipótese nula, o prêmio de risco não é simétrico para mercado em alta e em baixa.

Conclui-se que a existência de um tradeoff risco/rentabilidade positivo, confirmado por Pettengill, Sundaram e Mathur (1995) não existe na amostra em análise.

\section{CONCLUSÕES}

O objetivo desta investigação é analisar a capacidade explicativa do coeficiente beta nas ações cotadas no mercado português. Pettengill, Sundaram e Mathur (1995) constatam existir uma relação entre beta e rentabilidade, mas condicional com a evolução do mercado, assim como também

TABELA 5 - Estatísticas Descritivas da Rentabilidade em Excesso de Mercado

\begin{tabular}{lcccc}
\hline & \multicolumn{4}{c}{ Rendibilidade em Excesso de Mercado - Rme $=$ Rm-Rf } \\
\cline { 2 - 5 } Período: & Mean & Std Dev & T-statistic & $P$-value \\
\hline Período Total (01/09 a 12/16) & 0,0059 & 0,0062 & 0,9636 & 0,3381 \\
\hline Período 1 (01/09 a 12/12) & $-0,0072$ & 0,0078 & $-0,9214$ & 0,3622 \\
\hline Período 2 (01/13 a 12/16) & $\mathbf{0 , 0 1 9 1}$ & 0,0091 & 2,0844 & 0,0434 \\
\hline
\end{tabular}

Fonte:

O p-value corresponde ao teste estatístico da hipótese nula a média da Rme ser igual a zero.

As médias estatisticamente diferentes de zero, a um nível de significância de $5 \%$, estão assinaladas em negrito.

TABELA 6 - Relação de Simetria dos Coeficientes do Modelo com Beta Condicional

\begin{tabular}{cccccc}
\hline \multicolumn{5}{c}{ Média das Regressões: $R_{j, t}=\gamma_{0}+\gamma_{2} D_{t} \beta_{j-1}+\gamma_{3}\left(1-D_{t}\right) \beta_{j-1}+\varepsilon_{j, t}$} \\
\hline & $\mathrm{H}_{0}: \bar{\gamma}_{2}-\bar{\gamma}_{3}=0$ & \\
\hline $\begin{array}{c}\text { Período Total } \\
(01 / 2009 \text { a 12/2016) }\end{array}$ & \multicolumn{2}{c}{$\begin{array}{c}\text { Período 1 } \\
\text { Período 2 }\end{array}$} \\
\hline T-statistic & P-value & T-statistic & P-value & T-statistic & $P$-value \\
\hline 3,274 & 0,002 & 2,409 & 0,025 & 2,998 & 0,007 \\
\hline
\end{tabular}

Fonte: 
confirmam a existência de um tradeoff risco/rentabilidade positivo.

As conclusões obtidas para as ações cotadas na Euronext Lisboa entre janeiro/I999 a dezembro/2016, com base numa metodologia similar à utilizada por Pettengill, Sundaram e Mathur (1995) são:

(I) Não existe uma relação não condicional e positiva entre beta e rentabilidade. Resultado que é consistente entre períodos.

(2) Não existe uma relação condicional entre beta e rentabilidade. Quando o mercado está em alta não é confirmada a existência de uma relação positiva, resultado que é consistente entre períodos. Quando o mercado está em baixa é confirmada a existência de uma relação negativa entre beta e rentabilidade, mas inconsistente ao longo do período em análise.
(3) Não existe um tradeoff risco/rentabilidade positivo. No período dois a média da rentabilidade em excesso de mercado é negativa e, em todo o período da amostra o prêmio de risco para mercado em alta e em baixa não é simétrico.

A relação condicional entre beta e rentabilidade descrita por Pettengill, Sundaram e Mathur (1995) e confirmada posteriormente em vários estudos nomeadamente Durand, Lan e Ng (20I I), Fletcher (2000), Jianhua e Wihlborg (20I0), Morelli (20II) e, Theriou, Aggelidis, Maditinos et al. (2010) não é confirmada nas ações cotadas na Euronext Lisboa.

Dos resultados obtidos conclui-se que o coeficiente beta não é explicativo da rentabilidade das ações cotadas no mercado analisado. Em pesquisas futuras deve-se analisar a existência de fatores adicionais de risco. 


\section{REFERÊNCIAS}

ABDULLAH, K.; AL-JAFARI, M.; TAI, H.; AHMAD, A. The relationship between risk and return: An empirical study of Kuwait stock exchange. International Research Journal of Finance \& Economics, v. 66, p. 22-29, $201 \mathrm{l}$.

ANURADHA, N. The Conditional Relation between Beta and Returns: Evidence from Sri Lanka. Wayamba Journal of Management, v. 3, p. I-I2, 201 I.

BLACK, F.; JENSEN, M.; SCHOLES, $M$. The capital asset pricing model: Some empirical tests. Study in the Theory of Capital Markets, New York, M. C. Jensen, p. 79-121, 1972.

BLUME, M.; FRIEND, I. A new looks at the capital asset pricing model. Journal of Finance, v. 28, n. I, p. 19-33, 1973.

COTTER, J.; O'SULLIVAN, N.; ROSSI, F.The conditional pricing of systematic and idiosyncratic risk in the UK equity market. International Review of Financial Analysis, v. 27, p. 184-193, 2015.

CROMBEZ, J.; VENNET, R. Risk/return relationship conditional on market movements on the Brussels stock exchange. Tijdschrift voor Economie en Management, v. 45, n. 2, p. I63- I 88, 2000.

DURAND, R.; LAN, Y.; NG, A. Conditional beta: Evidence from Asian emerging. Global Finance Journal, v. 22, n. 2, p. I30-I53, 201 I.

ELSAS, R.; EL-SHAER, M.;THEISSEN, E. Beta and returns revisited: Evidence from the German stock market. Journal of International Financial Markets, Institutions and Money, v. I3, n. I, p. I- 18, 2003.

FAMA, E.; FRENCH, K. The cross-section of expected stock returns. Journal of Finance, v. 47, n. 2, p. 427-465, 1992.

FAMA, E.; MACBETH, J. Risk, return, and equilibrium: Empirical tests. Journal of Political Economy, v. 8I, n. 3, p. 607-636, 1973.

FLETCHER, J. On the conditional relationship between beta and return in international stock return. International Review of Financial Analysis, v. 9, n. 3, p. 235-245, 2000.

HUNG, D.; SHACKLETON, M.; XU, $X$. CAPM, higher co-moment and factor models of UK stock returns. Journal of Business Finance \& Accounting, v. 3 I, n. I/2, p. 87-II2, 2004.

ISAKOV, D. Is beta still alive? Conclusive evidence from the Swiss stock market. The European Journal of Finance, v. 5, n. 3, p. 202-212, 1999.

JAGANNATHAN, R.;WANG, Z. The conditional CAPM and the cross-section of expected returns. Journal of Finance, v. 5I, n. I, p. 3-53, 1996.

JIANHUA, Z.;WIHLBORG, C. CAPM in up and down markets: Evidence from six European emerging markets. Journal of Emerging Market Finance, v. 9, n. 2, p. 229255, 2010.

KASSIMATIS, K. Size, book to market and momentum effects in the Australian stock market. Australian Journal of Management, $v$. 33, n. I, p. I45- I68, 2008.

LAKONISHOK, J.; SHAPIRO, A. Systematic risk total risk and size as determinants of stock market returns. Journal of Banking \& Finance, v. I0, n. I, p. I I5- I32, 1986. LEWELLEN, J.; NAGEL, S. The conditional CAPM does not explain asset-pricing anomalies. Journal of Financial Economics, v. 82, n. 2, p. 289-3|4, 2006.

MORELLI, D. Joint conditionality in testing the beta-return relationship: Evidence based on the UK stock market. Journal of International Financial Markets, Institutions and Money, v. 2 I, n. I, p. I- I3, 20 I I.

NIMAL, P.; FERNANDO, S. The conditional relation between beta and returns: Evidence from Japan and Sri Lanka. The Hikone Ronso, Spring/395, p. 94-105, 2013.

PETTENGILL, G.; SUNDARAM, S.; MATHUR, I. The conditional relation between beta and returns. Journal of Financial and Quantitative Analysis, v. 30, n. I, p. I0I-I I6, I995.

REINGANUM, M. A new empirical perspective on the CAPM. Journal of Finance and Quantitative Analysis, v. 16, n. 4, p. 439-462, I98I. SANDOVAL,A.; SAENS, R. The conditional relationship between portfolio beta and return: Evidence from Latin America. Latin American Journal of Economics, v. 4I, p. 65-89, 2004.

TANG, G.; SHUM, W. The risk-return relations in the Singapore stock market. Pacific-Basin Finance Journal, v. I2, n. 2, p. I79- 195, 2004. TERREGROSSA, S.; ERASLAN, V. An analysis of the relation between return and beta for portfolios of Turkish equities. Cogent Economics \& Finance, v. 4, n. I, p. I-I2, 2016.

THERIOU, N.; AGGELIDIS, V.; MADITINOS, D.; ŠEVIC, Ž. Testing the relation between beta and returns in the Athens stock exchange. Managerial Finance, v. 36, n. 12 , p. 1043-I056, 2010.

TINIC, S.; WEST, R. Risk and return: January vs. the rest of the year. Journal of Financial Economics, v. I3, n. 4, p. 56I-574, 1984.

XIAO, B. Conditional Relationship Between Beta and Return in the US Stock Market. Expert Journal of Business and Management, v. 4, n. I, p. 46-55, 2016. 